\title{
A class of shot noise models for financial applications *柿
}

\author{
Gennady Samorodnitsky \\ Cornell University
}

January 17, 1996

\begin{abstract}
We describe a class of non-Markov shot noise processes that can be used as models for rates of return on securities, exchange rate processes and other processes in finance. These are continuous time processes that can exhibit heavy tails that become lighter when sampling interval increases, clustering and long memory.
\end{abstract}

\section{Introduction}

A "typical" stochastic process $\{Y(t), t \geq 0\}$ can be the log-price process of a particular security $S$ (a stock in particular), the log-exchange rate process between major currencies, etc. Understanding of the structure of the random process $\{Y(t), t \geq 0\}$ is of obvious importance, and many researchers in academia as well as those associated with the banking industry have taken a hard look on the data that accumulated throughout the years. Both the properties of the marginal distributions of the process and its dependence structure (in particular, correlations) have been thoroughly discussed, simply because those are the factors that affect the risk associated with the trading, and help in rational pricing of derivatives on securities (or on exchange rates). We begin with a discussion of some of the more important properties of the financial processes, which we formulate in terms of the return process (or increment process) $\{X(t)=Y(t+1)-Y(t), t \geq 0\}$. The latter process is usually regarded as being stationary (at

${ }^{*}$ This research was supported by the NSF grant DMS-94-00535, the NSA grant MDA904-95-H-1036 and United States - Israel Binational Science Foundation (BSF) Grant 92-00074/1 as well as by Forschungsinstitut für Mathematik and Institut für Operations Research of ETH, Zürich.

$\dagger_{\text {AMS }} 1991$ subject classification. Primary 60E07, 60G55, 62P05

$\ddagger$ Keywords and phrases: shot noise processes, non-Markov models, financial models, heavy tails, long-range dependence, regular variation, infinitely divisible processes, cluster Poisson point processes, Lévy measure, tail behavior of the distribution. 
least, within reasonable time scale). There is not universal consensus that financial data always exhibit all of these features. There is a growing empirical evidence, nevertheless, supporting the findings below.

\section{The marginal distribution of $X(t)$ is heavy tailed.}

This is always taken to mean that the tail probability $P(X(t)>\lambda)$ (and its counterpart on the negative side) is heavier than that of a normal distribution, and most often it is understood that this tail probability is regularly varying at infinity:

$$
P(X(t)>\lambda) \sim \lambda^{-\alpha} L(\lambda)
$$

for some $\alpha>0$, where $L$ is a slowly varying function (that is, $L(x y) / L(x) \rightarrow 1$ as $x \rightarrow \infty$ for every $y>0)$. Again, the same conclusion is reached for the left tail, $P(X(t)<-\lambda)$, as $\lambda \rightarrow \infty$ (Mandelbrot [Man63], Fama [Fam65], Blattberg and Gonedes [BG74], Mittnik and Rachev [MR93]). There is some evidence that the tail exponent $\alpha$ lies, in many cases, between 2 and 4 (Akgiray and Booth [AB88], Loretan and Phillipps [LP94]), even though there is still some debate whether the estimated tail exponent exceeding two truly rules

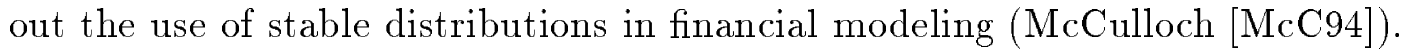

\section{The tails become less heavy as the sampling interval increases.}

That is, daily returns appear to have heavier tails than weekly returns which, in turn, appear to have heavier tails than monthly returns, etc. In other words, the tail exponent $\alpha$ in (1.1) increases when we add a certain number of consecutive observations. This was first reported in Akgiray and Booth [AB88]; see also Guillaume et al. [GDD*94] who report similar phenomena for high frequency data.

\section{Clustering in the data.}

This means that there are periods of high activity (and, thus, significant changes, or high volatility) and periods of low activity (low volatility). To say it differently, big changes come in clusters (Mandelbrot [Man63], Engle [Eng82]).

\section{Long-range dependence in the data.}

This means that $X(t)$ and $X(t+s)$ are highly dependent even for very large time lag $s$. This is most often observed through the sample autocorrelation function of the volatility (absolute changes) in the prices (Bollerslev, Chou and Kroner [BCK92] and also Guillaume et al. [GDD*94] in the high frequency context).

The following plots represent the daily returns from the $S \& P$ composite index for the period (July 2, 1962 - December 31, 1991), Hill estimator plots for the daily returns and 4-day returns, and the empirical autocorrelation function for the absolute returns.

The plot of the data set (Fig. 1.1) demonstrates the clustering phenomenon. The "catastrophe" at observations numbered around 6300-6400 corresponds to the market crash of October 1987. 
The Hill estimators of the tail exponents for the daily and 4-day returns are shown on Fig. 1.2. The reliability of this non-parametric technique for tail estimation is widely, though not universally, accepted.

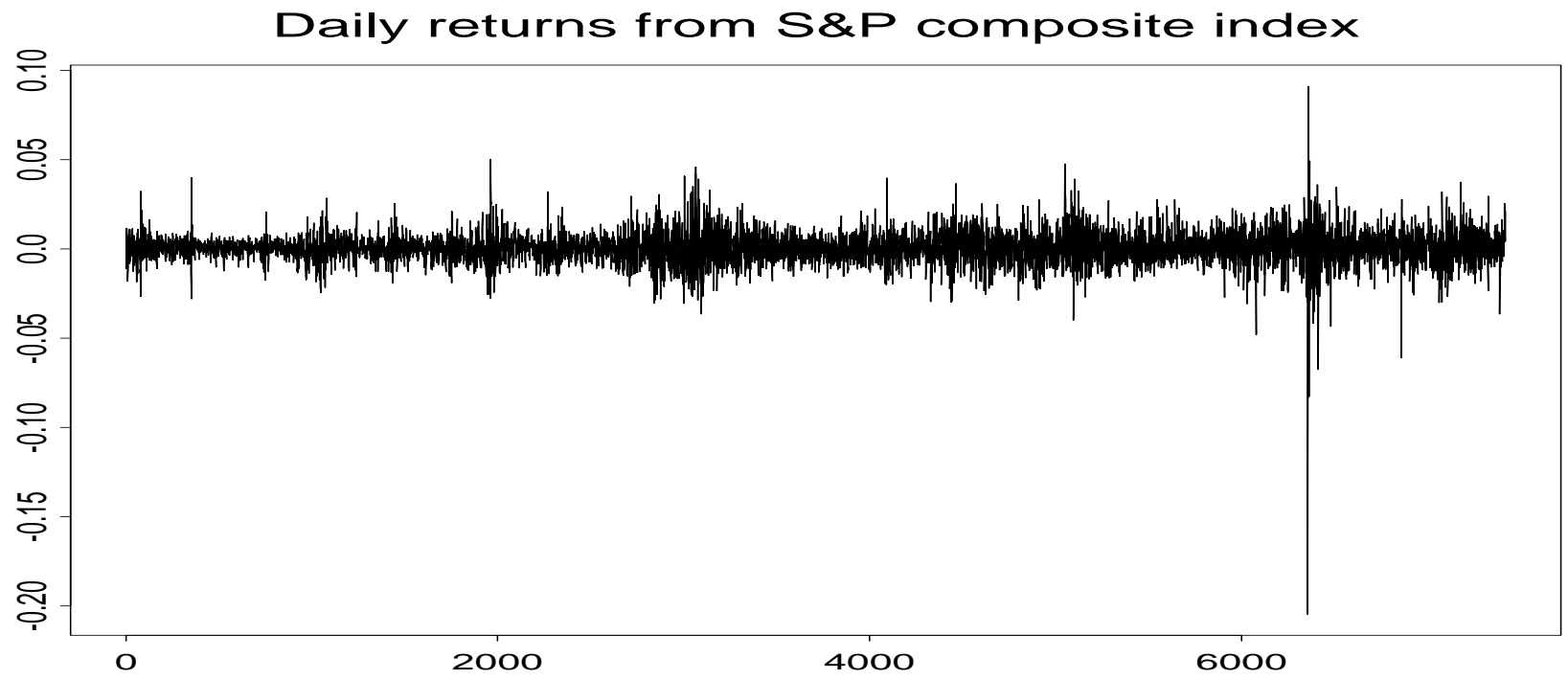

Figure 1.1

Hill estimator requires one to select the number of order statistics used in computing the estimator, and this is most often a difficult problem, typically solved in either rather heuristic way, or or by introducing certain assumptions on the actual marginal distribution of the daily returns.
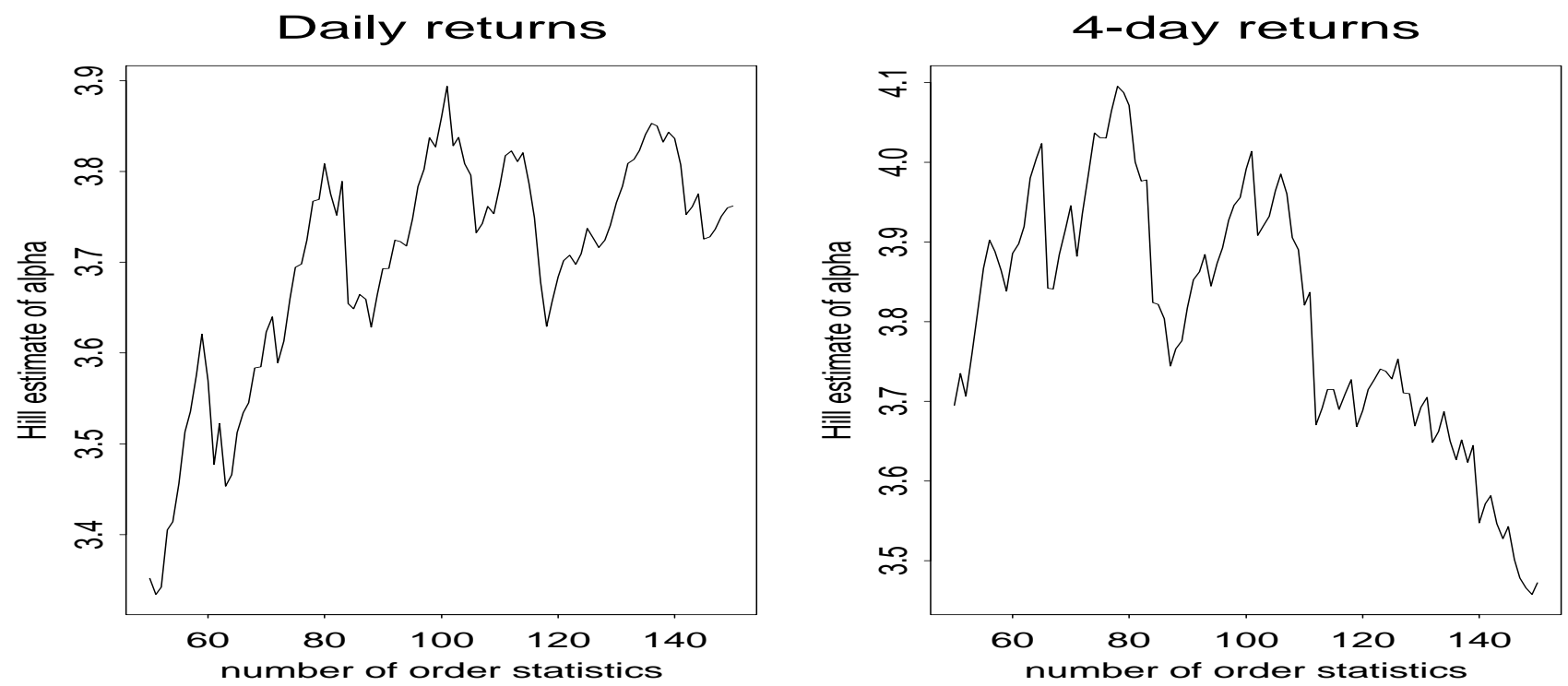

Figure 1.2 
The fact that the data under consideration is heavy tailed seems to be well established, and it is also possible to observe it by plotting the the tail of the empirical distribution function against its argument in the log-log coordinates. Presently we have selected a Hill estimator based on a relatively short sample (this tends to increase the variance but reduce the bias of the estimator). It seems to indicate an increase in the tail exponent from about $\alpha=3.75$ for daily returns to about $\alpha=3.95$ for 4 -day returns.

The empirical autocorrelation function depicted in Fig. 1.3 shows a very slow decay in correlations of absolute returns. It appears to be significant even after as much as 150-200 days.

Modeling of financial processes has developed, naturally, in two ways. The first is concerned with the dynamical structure of the processes, i.e. understanding how they develop in time. Here much work has been done along the lines of processes with stationary and independent increments ("the random walk hypothesis"), especially Brownian motion (Samuelson [Sam65], see also Föllmer and Schweizer [FS93] for a recent discussion), but also jump processes, like Lévy stable motion (Mandelbrot [Man63]) and others. Another body of work is devoted to $\mathrm{ARCH}$ and GARCH models, which are time series models that can account, for example, for the heavy tails and the clustering property of the financial processes.

Series : abs(nyse)

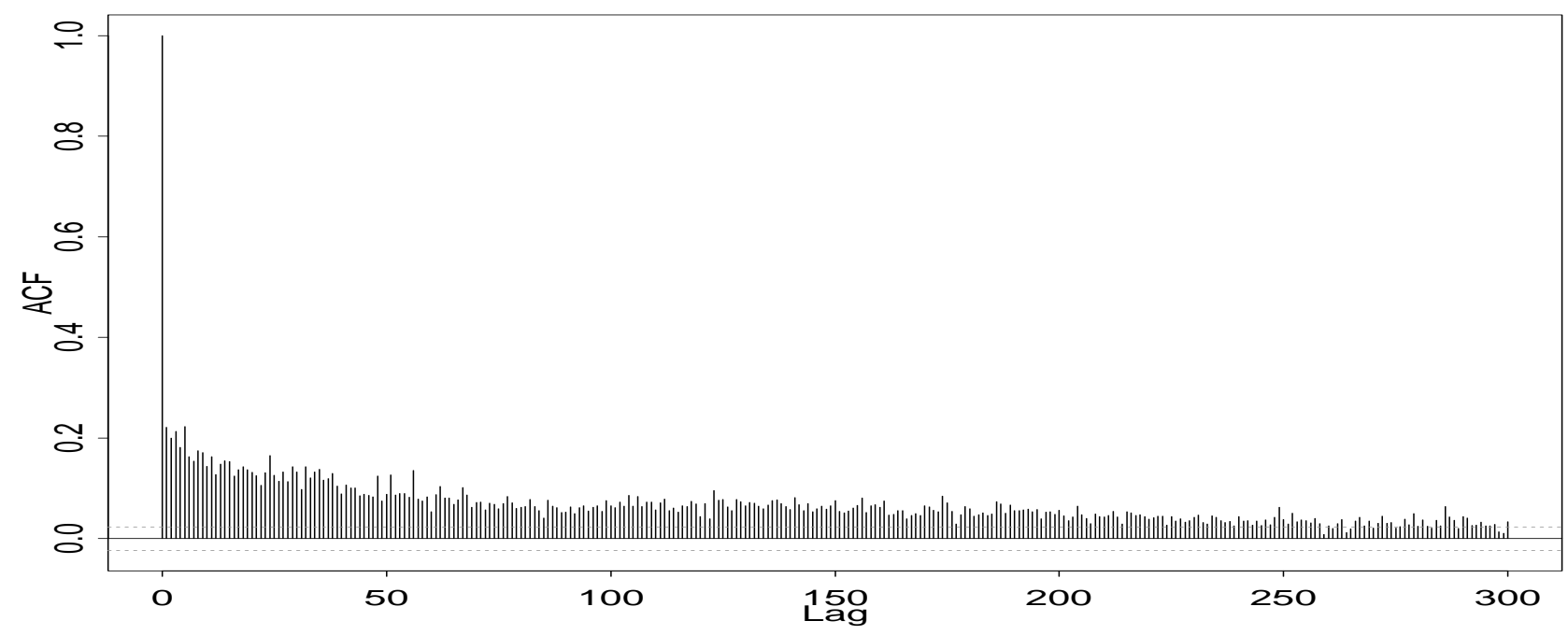

Figure 1.3

Secondly, it is important to find a good model for marginal distribution of the return $X(t)$, and there is a vast literature on the subject, beginning with the Gaussian and stable distributions mentioned above, up to very sophisticated families of distributions, showing an excellent fit to the data (alternative stable distributions of Mittnik and Rachev [MR93], hyperbolic distributions of Eberlein and Keller [EK94] and Küchler et al. [KNrS94], Student's $t$ distribution of Spanos [Spa93] and inverse Gaussian distribution of Barndorff-Nielsen [Bar94], to mention just a few). 
Of course, the above properties of financial processes show that the latter require careful modeling of both marginal distribution of $X(t)$ and the non-trivial dependence structure in the process $\{X(t), t \geq 0\}$. However, it is also important to have an intuitive model, one that seems to be in correspondence with the activity underlying the financial processes. For example, a process with stationary and independent increments has an obvious intuitive appeal by expressing the idea of "total randomness" of returns, even though the idea may not be universally accepted. See Taylor [Tay86] for a discussion. Stability (in a wide sense) property of financial models is another intuitive property, as emphasized by Mandelbrot [Man82] and Mittnik and Rachev [MR93].

The purpose of the present paper is to suggest a class of continuous time models for financial processes, the shot noise models, that arise naturally and intuitively if one thinks of the more significant activity in a market as caused by reaction of the market to various events (interest rate changes, mergers, announcements, expectations, rumors, political events, etc). Specifically, assume that the events affecting the market arrive at (random) times $T_{i}, i=1,2, \ldots$, the (random) initial effect of the event $i$ on the market is $Z_{i}$, and the this effect changes with time according to a deterministic function $f(t), t \geq 0$. The random variables $Z_{1}, Z_{2}, \ldots$ are assumed to be i.i.d. Since the effect of the event number $i$ begins at the time of its arrival, $T_{i}$, we can think of the return at time $t$ as the total of the effects caused up to this time, which gives

$$
X(t)=\sum_{T_{i} \leq t} Z_{i} f\left(t-T_{i}\right)
$$

Such a model is a simplification of the general shot noise model described usually as

$$
X(t)=\sum_{T_{i} \leq t} W_{i}\left(t-T_{i}\right)
$$

with i.i.d. stochastic processes $\left\{W_{i}(t), t \geq 0\right\}$. The deterministic nature of the dynamic effect of each event on the market leads to a simpler model (which by itself is very important), and we will argue in the sequel that even such a choice leads to a very rich class of models that can account for the properties of financial processes described above. One should mention that such processes are non-Markov moving averages, and that the many statistical problems associated with such processes are still being studied, and many others should yet be studied.

Shot noise processes are not a new phenomenon in probabilistic modeling. They are used in risk theory (Klüppelberg and Mikosch [KM93a] and [KM93b]), in modeling earthquake aftershocks (Vere-Jones [Ver70]) and computer failure times (Lewis [Lew64]), among others. We purport to show that these processes can be useful in financial modeling as well.

This paper is organized as follows. In the next section we define our model and discuss its basic properties. Section 3 contains a specific case of our model that exhibits many of the features of financial processes described in the present section, and Section 4 presents some simulation results and conclusions. 


\section{Shot noise processes}

We consider a stochastic process of the form

$$
X(t)=\sum_{i=1}^{\infty} Z_{i} f\left(t-T_{i}\right), t \in R
$$

where $f: R \rightarrow R$ is a measurable function vanishing on the negative half-line, $Z_{1}, Z_{2}, \ldots$ are i.i.d. random variables with a common distribution function $F$, and $T_{1}, T_{2}, \ldots$ constitute a cluster Poisson process independent of the sequence $Z_{1}, Z_{2}, \ldots$. Such a process is defined by three parameters: cluster arrival rate $\rho$, cluster size probabilities $p_{k}, k \geq 1$ and displacement distribution $H$. That is, cluster "centers" $C_{j}, j \geq 1$ arrive according to a time homogeneous Poisson process with rate $\rho$. The $j$ 'th cluster is of size $K_{j}=k$ with probability $p_{k}, k \geq 1$, and the points in this cluster are located at $C_{j}+D_{i j}, i=1, \ldots, K_{j}$. The array $\left\{D_{i j}, i \geq 1, j \geq 1\right\}$ is an array of i.i.d. random variables with common distribution $H$, independent of the two independent sequences: that of Poisson arrivals $\left\{C_{j} j \geq 1\right\}$ and that of i.i.d. discrete random variables $\left\{K_{j}, j \geq 1\right\}$ with probabilities given by $p_{k}, k \geq 1$.

We refer to the stochastic process $\{X(t), t \in R\}$ as a cluster Poisson shot noise process. In the particular case when all clusters are of size 1 with probability $1\left(p_{1}=1\right)$, the underlying point process $T_{1}, T_{2}, \ldots$ is just a time homogeneous Poisson process with rate $\rho$, leading to a simpler, Poisson shot noise model. The cluster nature of the more general model can, however, be useful for modeling the clustering property of financial processes.

One can see immediately from the definition of the cluster Poisson shot noise process that it suffers from an unidentifiability problem. Indeed, two "whole distributions" must be specified. In future work we plan to restrict the modeling to particular parametric classes of distributions, which can eliminate the unidentifiability problem. In certain cases one may want to decide on the form of the cluster size and displacement distributions from other considerations, based on the nature (or theories) of shocks affecting the market. It is this possibility of concentrating on the nature of the financial process that adds appeal to the proposed class of models.

We would like to emphasize from the beginning that, in spite of the deterministic nature of the function $f$ in (2.1), the shot noise process $\{X(t), t \in R\}$ is not predictable between the jumps $T_{i}, i=1,2, \ldots$, because the shots of the noise overlap. Therefore, observing the sample path of the process until a time $s$, say, it is not generally possible to infer the locations $T_{i}$ 's and the magnitudes $Z_{i}$ 's of the jumps up to that time, and so it is impossible to predict the behavior of the process until the next jump. It is this feature of shot noise processes that makes them valuable as stochastic models.

The Poisson character of a cluster Poisson process implies immediately that the cluster Poisson shot noise process (2.1) is infinitely divisible, whenever it is well defined (that is, whenever it is defined by a convergent sum). We would like to understand its properties, for which purpose it is more convenient to regard this process as a stochastic integral

$$
X(t)=\int_{R} f(t-s) M(d s), t \in R
$$


with respect to an cluster compound Poisson random measure $M$ defined by

$$
M(A)=\sum_{j=1}^{\infty} \sum_{i=1}^{K_{j}} Z_{i j} \mathbf{1}\left(\left(C_{j}+D_{i j}\right) \in A\right) .
$$

Here we have transformed, for obvious convenience, the i.i.d. sequence $Z_{1}, Z_{2}, \ldots$ into an i.i.d. array $Z_{i j}, i, j \geq 1$, with the same common distribution $F$. This form of representation of the cluster Poisson shot noise model shows that it is also a moving average with respect to the random measure $M$. The result of the following lemma is, undoubtedly, known. Nonetheless, it does not seem to be readily available, and we include it here for completeness. Notice that the lemma does not require the function $f$ to vanish on the negative half-line.

Lemma 2.1 Suppose that

$$
\int_{R} \sum_{k=1}^{\infty} p_{k} E\left(1 \wedge\left|\left(\sum_{i=1}^{k} Z_{i 1} f\left(x+D_{i 1}\right)\right)\right|\right) d x<\infty .
$$

Then the stochastic integral $I(f)=\int_{R} f(s) M(d s)$ defined by

$$
I(f)=\lim _{R \rightarrow \infty} \int_{R} f(s) M_{R}(d s):=\lim _{R \rightarrow \infty} I_{R}(f)
$$

where

$$
M_{R}(A)=\sum_{\left|C_{j}\right| \leq R} \sum_{i=1}^{K_{j}} Z_{i j} \mathbf{1}\left(\left(C_{j}+D_{i j}\right) \in A\right),
$$

exists as an almost sure limit, and is an infinitely divisible random variable with characteristic function

$$
E e^{i \theta I(f)}=\exp \left\{-\rho \int_{R}\left[1-\psi_{K}\left(E e^{i \theta Z_{11} f\left(x+D_{11}\right)}\right)\right] d x\right\}
$$

where $\psi_{K}(s)=\sum_{k=1}^{\infty} p_{k} s^{k},|s| \leq 1$ is the generating function of the cluster size $K$. Moreover, the Lévy measure of $I(f)$ is given by

$$
\mu=\rho \sum_{k=1}^{\infty} p_{k} \mu_{k}
$$

where for $k \geq 1$ the measure $\mu_{k}$ is defined by

$$
\mu_{k}=(L e b \otimes P) \circ T_{k}^{-1} .
$$

Here $T_{k}$ is a map from $R \times \Omega$ to $R$ defined by

$$
T_{k}(x, \omega)=\sum_{i=1}^{k} Z_{i 1}(\omega) f\left(x+D_{i 1}(\omega)\right), x \in R, \omega \in \Omega .
$$


Proof: The required computation can be done in a number of ways, the most "logical" of which is to "mark" the Poisson process by the clusters. Computationally, nonetheless, the easiest way seems to be the following direct argument.

Recalling the definition of the random measure $M$, we condition on the number of Poisson points in $(-R, R)$ whose locations are then uniformly distributed in $(-R, R)$ and conclude that

$$
\begin{gathered}
E e^{i \theta I_{R}(f)}=\sum_{n=0}^{\infty} e^{-2 \rho R} \frac{(2 \rho R)^{n}}{n !}\left(E e^{i \theta S(f)}\right)^{n} \\
=\exp \left\{-2 \rho R\left(1-E e^{i \theta S(f)}\right)\right\}
\end{gathered}
$$

where

$$
S(f)=\sum_{i=1}^{K_{1}} Z_{i 1} f\left(R U+D_{i 1}\right),
$$

and $U$ is a uniform random variable in $(-1,1)$ independent of the rest of the random variables above. It follows that

$$
E e^{i \theta S(f)}=\frac{1}{2 R} \int_{-R}^{R} \sum_{k=1}^{\infty} p_{k}\left(E e^{i \theta Z_{11} f\left(x+D_{11}\right)}\right)^{k} d x
$$

and therefore,

$$
E e^{i \theta I_{R}(f)}=\exp \left\{-\rho \int_{-R}^{R}\left[1-\psi_{K}\left(E e^{i \theta Z_{11} f\left(x+D_{11}\right)}\right)\right] d x\right\}
$$

From here we observe that the Lévy measure $\mu_{R}$ of $I_{R}(f)$ is given by (2.6), but the domains of the maps $T_{k}$ 's in (2.7) are restricted to $(-R, R) \times \Omega$. As $R \rightarrow \infty$ the measures $\mu_{R}$ increase to the measure $\mu$ given by (2.6). It follows easily from (2.4) that the characteristic function of $I_{R}(f)$ converges, as $R \rightarrow \infty$, to the expression in the right hand side of (2.5). Therefore, $I(f)$ exists as a limit in distribution, and its Lévy measure is given by (2.6). Finally, existence of $I(f)$ as an almost sure limit follows from the fact that, for a series of independent random variables, convergence in distribution is equivalent to the a.s. convergence.

Remark Note that when the compounding distribution $F$ is symmetric, the same argument shows that the integrability condition (2.4) may be replaced with a weaker condition

$$
\int_{R} \sum_{k=1}^{\infty} p_{k} E\left(1 \wedge\left|\left(\sum_{i=1}^{k} Z_{i 1} f\left(x+D_{i 1}\right)\right)\right|^{2}\right) d x<\infty .
$$

The assumption of homogeneous arrivals for the underlying Poisson process clearly implies that, once the function $f$ satisfies (2.4), the well defined stochastic process $\{X(t), t \geq 0\}$ given by $(2.1)$ or $(2.2)$ is a stationary process. Its second-order properties are described by the following lemma.

LEMmA 2.2 Let (2.4) hold, and assume that $E K_{1}^{2}<\infty, E Z_{11}^{2}<\infty$ and

$$
\int_{R} f(y)^{2} d y<\infty .
$$


Then the stochastic process $\{X(t), t \geq 0\}$ has a finite second moment, and its covariance function is given by

$$
R(t)=\rho E K_{1} E Z_{11}^{2} \int_{R} f(y) f(y+t) d y+\rho\left(E K_{1}^{2}-E K_{1}\right)\left(E Z_{11}\right)^{2} \delta(t),
$$

where

$$
\delta(t)=\int_{R} f(y+t)\left(\int_{R} \int_{R} f\left(y+x_{1}-x_{2}\right) H\left(d x_{1}\right) H\left(d x_{2}\right)\right) d y
$$

Proof: The fact that the process has a finite second moment follows from (2.6) and the fact that $E I(f)^{2}<\infty$ if and only if $\int_{R} x^{2} \mu(d x)<\infty$, and (2.10) follows directly from (2.1) by using repeatedly the conditioning argument

$$
\operatorname{cov}(X, Y)=E(\operatorname{cov}(X, Y \mid \mathcal{F}))+\operatorname{cov}(E(X \mid \mathcal{F}), E(Y \mid \mathcal{F}))
$$

\section{The process $X_{\gamma, d, m}$}

In this section we show that one can account for the properties exhibited by the financial processes and described in Section 1 by simple processes of the the shot noise type described in the previous section. We would like to emphasize that what we present here is only an example that exhibits these properties, and that many other cluster shot noise processes will share the latter. Let us take the compounding distribution $F$ to be the Laplace distribution. That is, $F$ is absolutely continuous with respect to Lebesgue measure, with the density

$$
F^{\prime}(x)=.5 e^{-|x|}, x \in R .
$$

The choice of the Laplace density, though fairly arbitrary, leads to simpler computations, and permits one to view probability tails through Laplace transform-type of lenses (c.f. (3.9)) and to utilize Tauberian-type arguments.

For a $\gamma>0, d>0$ and an integer $m \geq 1$ let

$$
f_{\gamma, d, m}(s)=j^{\gamma} \cos \pi \frac{j}{m} \text { if } s \in\left(j-(j+1)^{-(d+1)}, j+(j+1)^{-(d+1)}\right), j \geq 1,
$$

and $f_{\gamma, d, m}(s)=0$ is $s$ does not belong to any of the above intervals. Define

$$
X_{\gamma, d, m}(t)=\int_{R} f_{\gamma, d, m}(t-s) M(d s), t \geq 0,
$$

where $M$ is a cluster compound Poisson random measure. The following theorem describes the properties of the process $X_{\gamma, d, m}$. 
Theorem 3.1 (i) Suppose that $E K_{1}<\infty$. Then $X_{\gamma, d, m}$ is a well defined stationary stochastic process.

(ii) Assume that

$$
E K_{1}^{2}<\infty
$$

and that the displacement distribution $H$ is absolutely continuous with respect to the Lebesgue measure. Then

$$
P\left(X_{\gamma, d, m}(t)>\lambda\right) \sim c_{d, \gamma, m}^{(1)} \rho E K_{1} \lambda^{-d / \gamma}, \lambda \rightarrow \infty
$$

where

$$
c_{d, \gamma, m}^{(1)}=\frac{1}{2 \gamma m} \Gamma(d / \gamma) \sum_{i=0}^{2 m-1}\left|\cos \pi \frac{i}{m}\right|^{d / \gamma} .
$$

(iii) Under the assumptions of (ii), if $\gamma>1$, then

$$
P\left(\sum_{k=0}^{2 m-1} X_{\gamma, d, m}(t+k)>\lambda\right) \sim \begin{cases}c_{d, \gamma, m}^{(2)} \rho E K_{1} \lambda^{-(d+1) / \gamma} & \text { if } \frac{d+1}{\gamma}<\frac{d}{\gamma-1} \\ c_{d, \gamma, m}^{(3)} \rho E K_{1} \lambda^{-d /(\gamma-1)} & \text { if } \frac{d+1}{\gamma}>\frac{d}{\gamma-1} \\ \left(c_{d, \gamma, m}^{(2)}+c_{d, \gamma}^{(3)}\right) \rho E K_{1} \lambda^{-(d+1) / \gamma} & \text { if } \frac{d+1}{\gamma}=\frac{d}{\gamma-1}\end{cases}
$$

and if $\gamma \leq 1$ then

$$
P\left(\sum_{k=0}^{2 m-1} X_{\gamma, d, m}(t+k)>\lambda\right) \sim c_{d, \gamma, m}^{(2)} \rho E K_{1} \lambda^{-(d+1) / \gamma}
$$

as $\lambda \rightarrow \infty$. Here

$$
c_{d, \gamma, m}^{(2)}=\frac{1}{m} \Gamma((d+\gamma+1) / \gamma) \sum_{i=1}^{2 m-1} \sum_{j=1}^{2 m}\left|\sum_{k=0}^{i-1} \cos \pi(j+k) / 2 m\right|^{(d+1) / \gamma}>0
$$

and

$$
c_{d, \gamma, m}^{(3)}=\frac{1}{\gamma-1}(\gamma m)^{d /(\gamma-1)} \Gamma(d /(\gamma-1)) .
$$

(iv) Assume that (3.3) holds, and that

$$
d>2 \gamma
$$

Then the process $X_{\gamma, d, m}$ has a finite variance, and its covariance function $R(n)$ satisfies, as $n \rightarrow \infty$,

$$
R(n) \sim \kappa_{d, \gamma, m} \rho E K_{1} n^{2 \gamma-d} \sum_{i=1}^{2 m} \cos \pi i / m \cos \pi(i+n) / m
$$

in the sense that the product $n^{d-2 \gamma} R(n)$ has at most $2 m$ subsequential limits given by $(3.8)$, all of which are finite, and some of which are positive. Here

$$
\kappa_{d, \gamma, m}=2 m^{-1} \int_{0}^{\infty} x^{\gamma}(1+x)^{\gamma-d-1} d s
$$


Remark Note that the the probability tails of the process $X_{\gamma, d, m}$ are heavy, and that the tail of $\sum_{k=0}^{2 m-1} X_{\gamma, d, m}(k)$ is lighter than that $X_{\gamma, d, m}(0)$, which corresponds to the observed properties of financial processes. Secondly, the covariance function of $X_{\gamma, d, m}$ decays like a power, and not as exponential, function, and, if $2 \gamma<d \leq 2 \gamma+1$, then

$$
\sum_{n=1}^{\infty}|R(n)|=\infty
$$

that is, $X_{\gamma, d, m}$ exhibits long range dependence in its usual meaning (Brockwell and Davis [BD91]).

Proof of Theorem 3.1: Throughout the proof we will suppose, for simplicity of notation, that $\rho=1$.

(i) We need to check (2.9). Observe that, for a fixed $k$ and fixed displacements $D_{11}, \ldots, D_{k 1}$

$$
\left\{x \in R: \sum_{i=1}^{k} Z_{i 1} f\left(x+D_{i 1}\right) \neq 0\right\} \subset \bigcup_{i=1}^{k}\left(\mathcal{D}-D_{i 1}\right),
$$

where $\mathcal{D}$ is the domain of the function $f$ :

$$
\mathcal{D}=\bigcup_{j=1}^{\infty}\left(j-(j+1)^{-(d+1)}, j+(j+1)^{-(d+1)}\right) .
$$

Using the translation invariance of the Lebesgue measure we conclude that for any $k \geq 1$,

$$
\int_{R} E\left(1 \wedge\left|\left(\sum_{i=1}^{k} Z_{i 1} f\left(x+D_{i 1}\right)\right)\right|^{2}\right) d x \leq 2 k \sum_{j=1}^{\infty}(j+1)^{-(d+1)}
$$

and so (2.9) follows from $E K_{1}<\infty$ and $d>0$.

(ii) Suppose first that $K_{1}=1$ with probability 1 . Then $M$ is just a compound Poisson random measure, and we may assume that $D_{11}=0$ with probability 1 .

It follows from Lemma 2.1 that for any $\lambda>0$, the Lévy measure $\mu$ of $X_{\gamma, d, m}(t)$ satisfies

$$
\begin{gathered}
\mu\{(\lambda, \infty)\}=\frac{1}{2} \int_{-\infty}^{\infty} \int_{0}^{\infty} \mathbf{1}(x f(s)>\lambda) e^{-x} d x d s+\frac{1}{2} \int_{-\infty}^{\infty} \int_{0}^{\infty} \mathbf{1}(x f(s)<-\lambda) e^{-x} d x d s \\
=\frac{1}{2} \int_{0}^{\infty} \int_{0}^{\infty} \mathbf{1}(x|f(s)|>\lambda) e^{-x} d x d s \\
=\frac{1}{2} \int_{0}^{\infty} \exp (-\lambda /|f(s)|) d s .
\end{gathered}
$$

Substituting (3.1) into (3.9) we conclude that

$$
\mu\{(\lambda, \infty)\}=\sum_{j=1}^{\infty}(j+1)^{-(d+1)} \exp \left(-\lambda j^{-\gamma} /\left|\cos \pi \frac{j}{m}\right|\right)
$$




$$
\begin{gathered}
=\sum_{i=0}^{2 m-1} \sum_{j=1}^{\infty}(2 j m+i+1)^{-(d+1)} \exp \left(-\lambda(2 j m+i)^{-\gamma} /\left|\cos \pi \frac{i}{m}\right|\right) \\
:=\sum_{i=0}^{2 m-1} T_{i}(\lambda) .
\end{gathered}
$$

As $\lambda \rightarrow \infty$,

$$
T_{i}(\lambda) \sim \frac{1}{2 \gamma m} \Gamma(d / \gamma)\left|\cos \pi \frac{i}{m}\right|^{d / \gamma} \lambda^{-d / \gamma}
$$

thus proving that

$$
\mu\{(\lambda, \infty)\} \sim c_{d, \gamma, m}^{(1)} \lambda^{-d / \gamma}, \lambda \rightarrow \infty .
$$

We remark that in the case $K_{1}=1$ a.s. (3.4) already follows from (3.11) and the equivalence of the tail of an infinitely divisible random variable with that of its Lévy measure when the latter is subexponential (Theorem 1 of Embrechts, Goldie and Veraverbeke [EGV79]).

In the general case we use (2.6) and appeal to Theorem 3.2 with $\Phi(y, x)=y f(x)$ to conclude that

$$
\begin{gathered}
\mu\{(\lambda, \infty)\} \sim E K_{1} \mu_{1}\{(\lambda, \infty)\} \\
\sim c_{d, \gamma, m}^{(1)} E K_{1} \lambda^{-d / \gamma}, \lambda \rightarrow \infty
\end{gathered}
$$

which establishes (3.4) in the same way as above.

(iii) We already know from the proof of part (ii) that it is enough to prove our statement in the case $K_{1}=1$ and $D_{11}=0$ a.s.

The argument of (3.9) shows that for any $\lambda>0$ the Lévy measure $\mu$ of $\sum_{k=0}^{2 m-1} X_{\gamma, d, m}(t+k)$ is given by

$$
\mu\{(\lambda, \infty)\}=\frac{1}{2} \int_{R} \exp \left(-\lambda /\left|\sum_{k=0}^{2 m-1} f(s+k)\right|\right) d s .
$$

For $j \geq 1$ and $i=1,2, \ldots, 2 m$ we denote

$$
I_{i}^{(j)}=\left(j-(j+i)^{-(d+1)}, j+(j+i)^{-(d+1)}\right) .
$$

Then

$$
\begin{gathered}
\int_{R} \exp \left(-\lambda /\left|\sum_{k=0}^{2 m-1} f(s+k)\right|\right) d s=\int_{-(2 m-1)}^{1-2^{-(d+1)}} \exp \left(-\lambda /\left|\sum_{k=0}^{2 m-1} f(s+k)\right|\right) d s \\
+\sum_{j=1}^{\infty} \sum_{i=1}^{2 m-1} \int_{I_{i}^{(j)}-I_{i+1}^{(j)}} \exp \left(-\lambda /\left|\sum_{k=0}^{2 m-1} f(s+k)\right|\right) d s \\
+\sum_{j=1}^{\infty} \int_{I_{2 m}^{(j)}} \exp \left(-\lambda /\left|\sum_{k=0}^{2 m-1} f(s+k)\right|\right) d s \\
:=S_{0}(\lambda)+\sum_{i=1}^{2 m-1} S_{i}(\lambda)+S_{2 m}(\lambda) .
\end{gathered}
$$


Observe first that $f$ is bounded from above on intervals bounded away from infinity. Therefore,

$$
S_{0}(\lambda)=o\left(e^{-c \lambda}\right), \lambda \rightarrow \infty, c>0
$$

Now,

$$
S_{2 m}(\lambda)=\sum_{j=1}^{\infty} 2(j+2 m)^{-(d+1)} \exp \left(-\lambda /\left|\sum_{k=0}^{2 m-1}(j+k)^{\gamma} \cos \pi \frac{j+k}{m}\right|\right)
$$

Let

$$
a_{j}=\left|\sum_{k=0}^{2 m-1}(j+k)^{\gamma} \cos \pi \frac{j+k}{m}\right|, j=1,2, \ldots
$$

It is a straightforward calculation that

$$
a_{j} \sim m \gamma j^{\gamma-1}, j \rightarrow \infty
$$

Now there are two possibilities. If $\gamma \leq 1$ then it follows from (3.15) and (3.16) that

$$
S_{2 m}(\lambda)=o\left(e^{-c \lambda}\right), \lambda \rightarrow \infty, c>0
$$

whereas if $\gamma>1$ then

$$
\begin{aligned}
S_{2 m}(\lambda) \sim & \sum_{j=1}^{\infty} 2(j+2 m)^{-(d+1)} \exp \left(-\lambda / m \gamma j^{\gamma-1}\right) \\
& \sim 2 c_{d, \gamma, m}^{(3)} \lambda^{-d /(\gamma-1)}, \lambda \rightarrow \infty
\end{aligned}
$$

It remains to consider the terms $S_{i}(\lambda), i=1, \ldots, 2 m-1$ in (3.13). For each $i$ as above we have

$$
S_{i}(\lambda)=2 \sum_{j=1}^{\infty}\left((j+i)^{-(d+1)}-(j+i+1)^{-(d+1)}\right) \exp \left(-\lambda /\left|\sum_{k=0}^{i-1}(j+k)^{\gamma} \cos \pi(j+k) / m\right|\right) .
$$

Denote

$$
b_{j}^{(i)}=\left|\sum_{k=0}^{i-1}(j+k)^{\gamma} \cos \pi(j+k) / m\right|, i=1, \ldots, 2 m-1, j=1,2, \ldots
$$

Now, for each $i=1, \ldots, 2 m-1$ and $r=1,2, \ldots, 2 m$

$$
\frac{b_{j}^{(i)}}{j^{\gamma}} \rightarrow\left|\sum_{k=0}^{i-1} \cos \pi \frac{r+k}{m}\right|:=L(i, m, r)
$$

as $j \rightarrow \infty$ along the subsequence $j=r+2 m J, J=1,2, \ldots$ Clearly, each $L(i, m, r)$ is a finite nonnegative number, and it is straightforward to check that for every fixed $i=1, \ldots, 2 m-1$ 
there is an $r=1,2, \ldots, 2 m$ such that $L(i, m, r)>0$. This means that if we let $\mathcal{L}^{(i)}=\{r=$ $1,2, \ldots, 2 m: L(i, m, r)>0\}$, then $\mathcal{L}^{(i)} \neq \emptyset$. Write for $i=1, \ldots, 2 m-1$

$$
S_{i}(\lambda)=\sum_{r=1}^{2 m-1} S_{i, r}(\lambda)
$$

where

$$
=2 \sum_{J=0}^{\infty}\left((2 m J+r+i)^{-(d+1)}-(2 m J+r+i+1)^{-(d+1)}\right) \exp \left(-\lambda /\left|\sum_{k=0}^{i-1}(2 m J+r+k)^{\gamma} \cos \pi \frac{r+k}{m}\right|\right) .
$$

For every $r \in \mathcal{L}^{(i)}$ we have, as $\lambda \rightarrow \infty$,

$$
S_{i, r}(\lambda) \sim \frac{d+1}{2 \gamma m} L(i, m, r)^{(d+1) / \gamma} \Gamma((d+1) / \gamma) \lambda^{-(d+1) / \gamma}
$$

while for every $r \notin \mathcal{L}^{(i)}$ we have, as $\lambda \rightarrow \infty$,

$$
S_{i, r}(\lambda)=o\left(\lambda^{-(d+1) / \gamma}\right)
$$

Therefore, for every $i=1, \ldots, 2 m-1$,

$$
S_{i}(\lambda) \sim 2 c_{d, \gamma, m}^{(2)} \lambda^{-(d+1) / \gamma} \lambda \rightarrow \infty .
$$

Now the conclusion of part (iii) of the theorem follows from (3.12), (3.13), (3.14), (3.17), (3.18), (3.20) and Theorem 1 of Embrechts, Goldie and Veraverbeke [EGV79].

(iv) Since

$$
\int_{R} f(y)^{2} d y=2 \sum_{j=1}^{\infty} j^{2 \gamma}(j+1)^{-(d+1)} \cos ^{2} \pi \frac{j}{m}<\infty
$$

under (3.7), we conclude from Lemma 2.2 that the process $X_{\gamma, d, m}$ has a finite variance. Moreover, it follows from (2.10) that for every $n \geq 0$,

$$
\begin{gathered}
R(n)=2 E K_{1} \int_{R} f(y) f(y+n) d y \\
=4 E K_{1} \sum_{j=1}^{\infty} j^{\gamma}(j+n)^{\gamma} \cos \pi \frac{j}{m} \cos \pi \frac{j+n}{m}(j+n+1)^{-(d+1)} \\
=4 E K_{1} \sum_{i=1}^{2 m} \cos \pi \frac{i}{m} \cos \pi \frac{i+n}{m} \sum_{j=0}^{\infty}(i+2 m j)^{\gamma}(i+2 m j+n)^{\gamma}(i+2 m j+n+1)^{-(d+1)} .
\end{gathered}
$$

Now, for each $i=1, \ldots, 2 m$ we have

$$
\sum_{j=0}^{\infty}(i+2 m j)^{\gamma}(i+2 m j+n)^{\gamma}(i+2 m j+n+1)^{-(d+1)} \sim n^{2 \gamma-d}(2 m)^{-1} \int_{0}^{\infty} x^{\gamma}(1+x)^{\gamma-d-1} d x
$$


as $n \rightarrow \infty$, and (3.8) follows. This completes the proof of the theorem.

The remainder of this section is devoted to the proof of an ingredient in the argument of Theorem 3.1. Moreover, it concerns a question which is interesting in its own right. Given a sequence of i.i.d. random variables $X_{1}, X_{2}, \ldots$ with a common subexponential distribution $F$ (that is, such that $\lim _{\lambda \rightarrow \infty} \frac{\overline{F * F}(\lambda)}{\bar{F}(\lambda)}=2$ ), it turns out that

$$
\lim _{\lambda \rightarrow \infty} \frac{\overline{F^{* m}}(\lambda)}{\bar{F}(\lambda)}=m
$$

for any $m \geq 1$ and, moreover, if $N$ is an independent of the i.i.d. sequence integer valued nonnegative random variable, then under quite general conditions we have

$$
\lim _{\lambda \rightarrow \infty} \frac{P\left(\sum_{i=1}^{N} X_{i}>\lambda\right)}{P\left(X_{1}>\lambda\right)}=E N .
$$

see Chover, Ney and Wainger [CNW73], Embrechts, Goldie and Veraverbeke [EGV79] and Cline [Cli87]. In short, taking convolution powers of subexponential distributions preserves their tails.

Suppose now that taking convolution powers is performed conditionally. That is, let $(A, \mathcal{A}, \eta)$ be a $\sigma$-finite measure space, and let $(F(x, \cdot), x \in A)$ be a measurable family of distributions on $R$ such that

$$
\bar{G}(\lambda):=\int_{A} F(x,(\lambda, \infty)) \eta(d x)<\infty
$$

for all $\lambda$ large enough, and such that the distribution function $G=1-\min (\bar{G}, 1)$ belongs to the subexponential class. For each $x \in A$ let $X_{i}^{x}, i \geq 1$ be a sequence of i.i.d. random variables with common distribution $F(x, \cdot)$. Under what conditions is it true that

$$
\lim _{\lambda \rightarrow \infty} \frac{\int_{A} P\left(\sum_{i=1}^{m} X_{i}^{x}>\lambda\right) \eta(d x)}{\int_{A} P\left(X_{1}^{x}>\lambda\right) \eta(d x)}=m
$$

for every $m \geq 1$, and, more generally, under what conditions is the counterpart of (3.21) true:

$$
\lim _{\lambda \rightarrow \infty} \frac{\int_{A} P\left(\sum_{i=1}^{N} X_{i}^{x}>\lambda\right) \eta(d x)}{\int_{A} P\left(X_{1}^{x}>\lambda\right) \eta(d x)}=E N
$$

when $N$ is independent of the sequence $X_{i}^{x}, i \geq 1$ ?

In this paper we consider only the case of a regularly varying $\bar{G}$, and the family $(F(x, \cdot), x \in$ $A)$ generated in a rather special way. However, one can see even here that new phenomena arise, those not present when one takes unconditional convolution powers.

Theorem $3.2 \operatorname{Let}(A, \mathcal{A}, \eta)=(R, \mathcal{B}$, Leb), and for an $x \in R$ let $F(x, \cdot)$ be the distribution of $\Phi\left(Y_{1}, D_{1}+x\right)$, where $\Phi: R^{2} \rightarrow R$ is a measurable function, and $\left(Y_{i}, i \geq 1\right)$ and $\left(D_{i}, i \geq 1\right)$ are independent sequences of i.i.d. random variables. 
Assume that the function $\bar{G}$ belongs to the class of regularly varying (at infinity) functions, with parameter $-p$ for some $p \geq 0$. Assume further that the distribution of $D_{1}$ is absolutely continuous with respect to the Lebesgue measure. Then (3.23) holds. More generally, if $N$ is an independent of the two i.i.d. sequences integer valued nonnegative random variable, with $E N^{2}<\infty$, then (3.24) holds as well.

Proof: The crucial step in the proof is the following statement.

$$
\lim _{\lambda \rightarrow \infty} \frac{\int_{R}\left[P\left(\Phi\left(Y_{1}, D_{1}+x\right)>\lambda\right)\right]^{2} d x}{\int_{R} P\left(\Phi\left(Y_{1}, D_{1}+x\right)>\lambda\right) d x}=0 .
$$

Suppose (3.25) has been proved. We have, for every $m \geq 2, \delta>0$ and $\lambda>0$ so big that (3.22) holds,

$$
\begin{gathered}
\int_{R} P\left(\sum_{i=1}^{m} \Phi\left(Y_{i}, D_{i}+x\right)>\lambda\right) d x \\
\geq m \int_{R} P\left(\Phi\left(Y_{1}, D_{1}+x\right)>\lambda(1+\delta)\right)\left[P\left(\Phi\left(Y_{1}, D_{1}+x\right) \leq \lambda \delta /(m-1)\right)\right]^{m-1} d x \\
=m \int_{R} P\left(\Phi\left(Y_{1}, D_{1}+x\right)>\lambda(1+\delta)\right) d x \\
-m \sum_{i=1}^{m-1}\left(\begin{array}{c}
m-1 \\
i
\end{array}\right)(-1)^{i-1} \int_{R} P\left(\Phi\left(Y_{1}, D_{1}+x\right)>\lambda(1+\delta)\right)\left[P\left(\Phi\left(Y_{1}, D_{1}+x\right)>\lambda \delta /(m-1)\right)\right]^{i} d x \\
:=m \bar{G}(\lambda(1+\delta))-R(\lambda) .
\end{gathered}
$$

Now, it follows from $(3.25)$ that

$$
|R(\lambda)| \leq m\left(2^{m-1}-1\right) \int_{R}\left[P\left(\Phi\left(Y_{1}, D_{1}+x\right)>\lambda \delta /(m-1)\right)\right]^{2} d x=o(\bar{G}(\lambda))
$$

as $\lambda \rightarrow \infty$. We conclude that

$$
\underline{\lim }_{\lambda \rightarrow \infty} \frac{\int_{R} P\left(\sum_{i=1}^{m} \Phi\left(Y_{i}, D_{i}+x\right)>\lambda\right) d x}{\bar{G}(\lambda)} \geq m(1+\delta)^{-p}
$$

and letting $\delta \rightarrow 0$ we obtain

$$
\underline{\lim }_{\lambda \rightarrow \infty} \frac{\int_{R} P\left(\sum_{i=1}^{m} \Phi\left(Y_{i}, D_{i}+x\right)>\lambda\right) d x}{\bar{G}(\lambda)} \geq m
$$

for every $m \geq 1$. Furthermore, it follows immediately from (3.26) and Fatou's lemma that

$$
\varliminf_{\lambda \rightarrow \infty} \frac{\int_{R} P\left(\sum_{i=1}^{N} \Phi\left(Y_{i}, D_{i}+x\right)>\lambda\right) d x}{\bar{G}(\lambda)} \geq E N
$$


whether or not $E N^{2}<\infty$.

For the upper bound, take an $m$ and $\lambda$ as before, and take also a $\delta \in(0,1)$. We have

$$
\begin{gathered}
\int_{R} P\left(\sum_{i=1}^{m} \Phi\left(Y_{i}, D_{i}+x\right)>\lambda\right) d x \\
\leq m \int_{R} P\left(\Phi\left(Y_{1}, D_{1}+x\right)>\lambda(1-\delta)\right) d x+\frac{m(m-1)}{2} \int_{R}\left[P\left(\Phi\left(Y_{1}, D_{1}+x\right)>\lambda \delta\right)\right]^{2} d x .
\end{gathered}
$$

From here, (3.25) and regular variation we get a matching upper bound to (3.26), thus proving (3.23). Furthermore,

$$
\begin{gathered}
\int_{R} P\left(\sum_{i=1}^{N} \Phi\left(Y_{i}, D_{i}+x\right)>\lambda\right) d x \\
\leq E N \bar{G}(\lambda(1-\delta))+E \frac{N(N-1)}{2} \int_{R}\left[P\left(\Phi\left(Y_{1}, D_{1}+x\right)>\lambda \delta\right)\right]^{2} d x,
\end{gathered}
$$

and using once again (3.25) and regular variation we obtain

$$
\varlimsup_{\lambda \rightarrow \infty} \frac{\int_{R} P\left(\sum_{i=1}^{N} \Phi\left(Y_{i}, D_{i}+x\right)>\lambda\right) d x}{\bar{G}(\lambda)} \leq E N(1-\delta)^{-p},
$$

from which (3.24) follows by letting $\delta \rightarrow 0$ and using (3.27).

It remains, therefore, to prove (3.25).

Assume first that the density $h$ of the distribution of $D_{1}$ with respect to the Lebesgue measure is bounded. That is, there is a $C<\infty$ such that $h(x) \leq C$ for every $x \in R$. We have then

$$
\begin{gathered}
\int_{R}\left[P\left(\Phi\left(Y_{1}, D_{1}+x\right)>\lambda\right)\right]^{2} d x \\
\left.=\int_{R}\left[P\left(\int_{R} \Phi\left(Y_{1}, x+y\right)>\lambda\right) h(y) d y \int_{R} \Phi\left(Y_{1}, x+z\right)>\lambda\right) h(z) d z\right] d x \\
\left.\left.=\int_{R} \Phi\left(Y_{1}, y\right)>\lambda\right) d y \int_{R} \Phi\left(Y_{1}, z\right)>\lambda\right) d z \int_{R} h(y-x) h(z-x) d x \\
\leq C\left[\int_{R} P\left(\Phi\left(Y_{1}, x\right)>\lambda\right) d x\right]^{2} \\
=C\left[\int_{R} P\left(\Phi\left(Y_{1}, D_{1}+x\right)>\lambda\right) d x\right]^{2},
\end{gathered}
$$

which proves (3.25) in that case.

We now remove the assumption of the boundedness of the density of $D_{1}$. For any $\epsilon>0$ one can find two random variables, $D_{1}^{\prime}$ and $D_{1}^{\prime \prime}$, such that $D_{1}^{\prime \prime}$ has a bounded density, and for every Borel set $A$,

$$
P\left(D_{1} \in A\right)=\epsilon P\left(D_{1}^{\prime} \in A\right)+(1-\epsilon) P\left(D_{1}^{\prime \prime} \in A\right) .
$$

We have by (3.28)

$$
\int_{R}\left[P\left(\Phi\left(Y_{1}, D_{1}+x\right)>\lambda\right)\right]^{2} d x
$$




$$
\begin{gathered}
=\int_{R}\left[\epsilon P\left(\Phi\left(Y_{1}, D_{1}^{\prime}+x\right)>\lambda\right)+(1-\epsilon) P\left(\Phi\left(Y_{1}, D_{1}^{\prime \prime}+x\right)>\lambda\right)\right]^{2} d x \\
\leq\left(\epsilon^{2}+2 \epsilon\right) \int_{R} P\left(\Phi\left(Y_{1}, D_{1}^{\prime}+x\right)>\lambda\right) d x+(1-\epsilon)^{2} \int_{R}\left[P\left(\Phi\left(Y_{1}, D_{1}^{\prime \prime}+x\right)>\lambda\right)\right]^{2} d x .
\end{gathered}
$$

Since (3.25) has already been proved for random variables $D_{1}$ that have a bounded density, we conclude that

$$
\varlimsup_{\lambda \rightarrow \infty} \frac{\int_{R}\left[P\left(\Phi\left(Y_{1}, D_{1}+x\right)>\lambda\right)\right]^{2} d x}{\int_{R} P\left(\Phi\left(Y_{1}, D_{1}+x\right)>\lambda\right) d x} \leq\left(\epsilon^{2}+2 \epsilon\right),
$$

from which (3.25) follows by letting $\epsilon \rightarrow 0$.

\section{Remarks}

1. The surprising fact is that the statement of Theorem 3.2 is false without some assumption of regularity of the distribution of the random variable $D_{1}$. It is false, for example, if the latter has atoms. To see this, consider the case $D_{1}=0$ a.s, $Y_{1}$ is exponential random variable with mean 1 , and $\Phi(y, x)=y|f(x)|$, with $f$ given by (3.1). Then

$$
\eta_{1}(\lambda):=\int_{R} P\left(\Phi\left(Y_{1}, D_{1}+x\right)>\lambda\right) d x=\int_{0}^{\infty} \exp (-\lambda /|f(x)|) d x
$$

is regularly varying at infinity with parameter $-p=-d / \gamma$. Furthermore,

$$
\begin{gathered}
\eta_{2}(\lambda):=\int_{R} P\left(\Phi\left(Y_{1}, D_{1}+x\right)+\Phi\left(Y_{2}, D_{2}+x\right)>\lambda\right) d x=\int_{0}^{\infty} \exp (-\lambda /|f(x)|)(1+\lambda /|f(x)|) d x \\
=\eta_{1}(\lambda)+\lambda \eta_{1}^{\prime}(\lambda) .
\end{gathered}
$$

It follows now from Proposition 0.7 of Resnick [Res87] that

$$
\lim _{\lambda \rightarrow \infty} \frac{\int_{R} P\left(\Phi\left(Y_{1}, D_{1}+x\right)+\Phi\left(Y_{2}, D_{2}+x\right)>\lambda\right) d x}{\int_{R} P\left(\Phi\left(Y_{1}, D_{1}+x\right)>\lambda\right) d x}=1+p
$$

which is, in general, different from 2.

2. We do not know whether the statement of Theorem 3.2 remains true if the distribution of $D_{1}$ is atomless, but not absolutely continuous with respect to the Lebesgue measure.

\section{Simulation of the process $X_{\gamma, d, m}$ and conclusions}

One can simulate the process $X_{\gamma, d, m}$ using directly the integral (or series) definition (2.2) and (2.3), truncating the sum over clusters by a certain finite number. The result of such a simulation is presented below. We emphasize that the whole problem of parameter estimation for a 
shot noise model is still under investigation, and the purpose of presenting a simulation result here is to give the reader an idea how a cluster shot noise process can behave.

Shot noise process

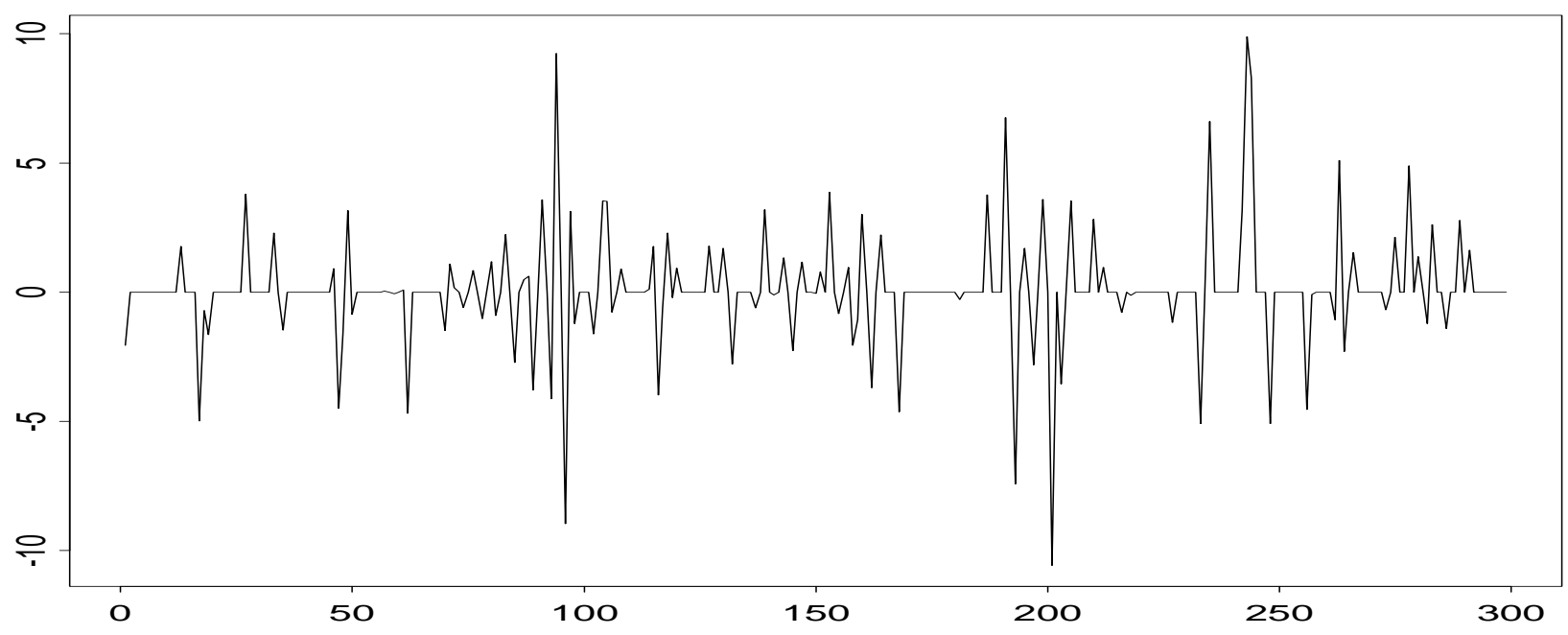

Figure 4.1

For the purpose of simulation we have selected the cluster size distribution to be geometric: $p_{k}=p(1-p)^{k-1}, k=1,2, \ldots$, with $p=.1$ (i.e. clusters have the average size of 10 ), the displacement distribution $H$ is uniform in the interval $(0, .3)$ and the cluster arrival rate is $\rho=1$. We have selected the parameters of the kernel $f_{\gamma, d, m}$ to be $m=2, d=.2$ and $\gamma=.5$, thus corresponding to the probability tails of $P\left(X_{\gamma, d, m}(0)>\lambda\right) \sim c \lambda^{-4}$ and $P\left(X_{\gamma, d, m}(0)+\right.$ $\left.X_{\gamma, d, m}(1)+X_{\gamma, d, m}(2)+X_{\gamma, d, m}(3)>\lambda\right) \sim c \lambda^{-6}$ as $\lambda \rightarrow \infty$. For the purpose of simulation we have taken 600 Poisson clusters.

The result of a simulation is presented on Fig. 4.1. Observe that the process $X_{\gamma, d, m}$ has a finite Lévy measure, so it will unavoidably take some zero values (this property can be actually useful in the context of high frequency data). The frequency with which zero values appear can be reduced by increasing the intensity of the point process $\rho$ and the cluster size $K_{1}$.

A possible point of view of a model having the above properties is to regard it as modeling the significant events taking place on the market, with the rest of activity being regarded as, say, a Brownian motion, or another simple noise. We have added to the above shot noise process an independent standard white noise, with the result appearing on Fig. 4.2. Heavy tails and clustering phenomena are highly visible in the two plots.

\section{Conclusions}

We have described a class of cluster Poisson shot noise models that can naturally exhibit heavy tails that get lighter under summation of consecutive observations, long memory and clustering. Those processes are non-Markov moving averages with respect to certain random measures. Problems of parameter estimation, prediction and derivative pricing have to be studied for such models, but it seems clear that their intuitive structure, and the ability to explain, in a parsimonious way, many characteristics of financial processes, make them quite 
an attractive class of models for the latter.

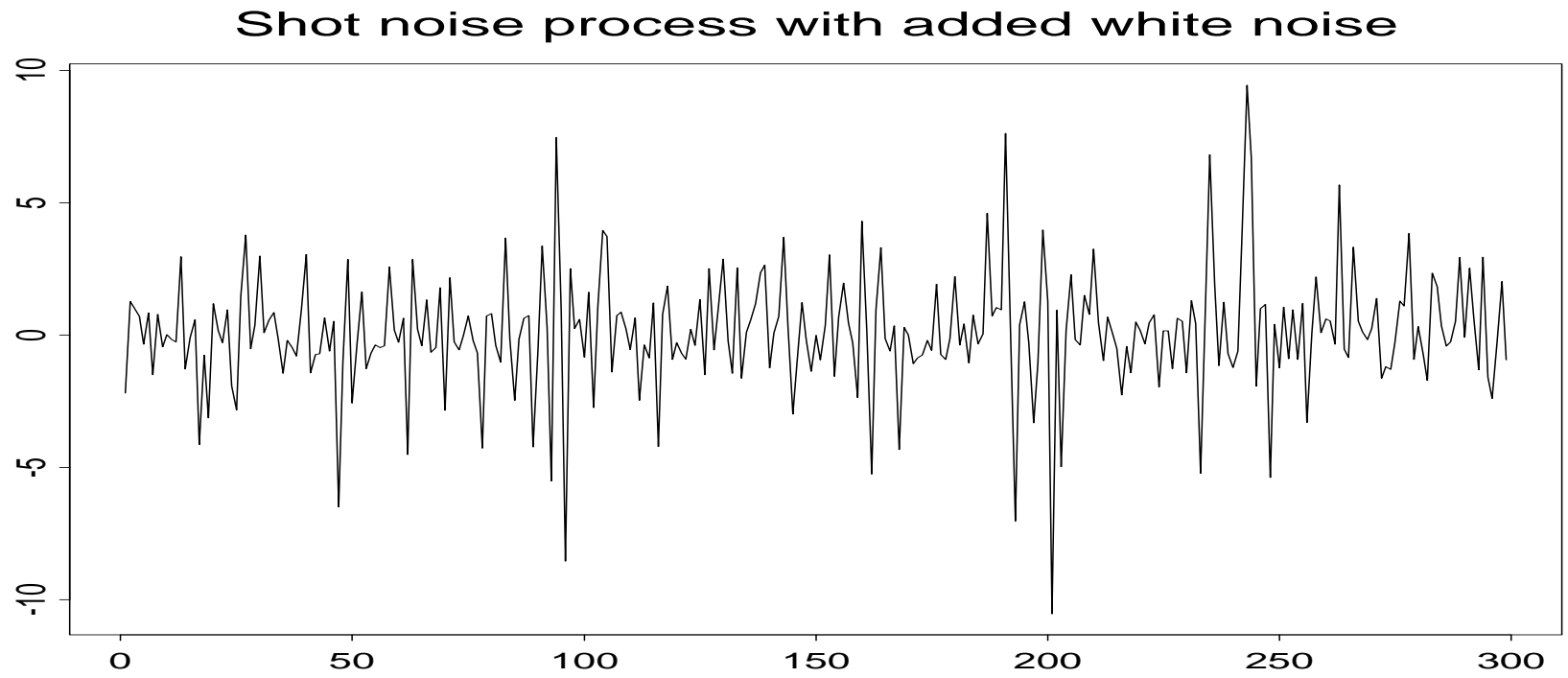

Figure 4.2

\section{References}

[AB88] V. Akgirav and G.G. Booth. The stable-law model of stock returns. J. Bus. Econ. Stat., 6:51-57, 1988.

[Bar94] O.E. Barndorff-Nielsen. Gaussian \\Inverse Gaussian processes and the modelling of stock returns. 1994. Technical report, Aarhus University.

[BCK92] T. Bollerslev, R.Y. Chow, and K.F. Kroner. ARCH modeling in Finance: a review of the theory and empirical evidence. Journal of Econometrics, 52:5-59, 1992.

[BD91] P.J. Brockwell and R.A. Davis. Time Series: Theory and Methods. Springer-Verlag, New York, second edition, 1991.

[BG74] R.C. Blattberg and N.J. Gonedes. A comparison of the stable and Student distributions as statistical models for stock prices. Journal of Business, 47:244-280, 1974.

[Cli87] D.B.H. Cline. Convolutions of distributions with exponential and subexponential tails. J. Austral. Math. Soc., 43:347-365, 1987. (Series A).

[CNW73] J. Chover, P. Ney, and S. Wainger. Functions of probability measures. J. Analyse Math., 26:255-302, 1973. 
[EGV79] P. Embrechts, C.M. Goldie, and N. Veraverbeke. Subexponentiality and infinite divisibility. Z. Wahr. verw. Geb., 49:335-347, 1979.

[EK94] E. Eberlein and U. Keller. Hyperbolic distributions in Finance. 1994. Technical report.

[Eng82] R.F. Engle. Autoregressive conditional heteroskedasticity with estimates of the variance of U.K. inflation. Econometrica, 50:987-1008, 1982.

[Fam65] E. Fama. The behavior of stock market prices. Journal of Business, 38:34-105, 1965.

[FS93] H. Föllmer and M. Schweizer. A microeconomic approach to diffusion models for stock prices. Math. Finance, 3:1-23, 1993.

[GDD*94] D.M. Guillaume, M.M. Dagorodna, R.R. Davé, U.A. Müller, R.B. Olsen, and O.V. Pictet. From the bird's eye to the microscope: a survey of new stylized facts of the intra-daily foreign exchange markets. 1994. A discussion paper by the O\& A Research Group.

[KM93a] C. Klüppelberg and T. Mikosch. Explosive Poisson shot noise processes with applications to risk reserves. 1993. Bernoulli, to appear.

[KM93b] C. Klüppelberg and T. Mikosch. Modelling delay in claim settlement. 1993. Scand. Actuar. Journal, to appear.

[KNrS94] U. Küchler, K. Neumann, M. Sørensen, and A. Streller. Stock returns and hyperbolic distributions. 1994. Technical report, Humboldt Universität zu Berlin.

[Lew64] P.A. Lewis. A branching Poisson process model for the analysis of computer failure pattern. Journal Royal Stat. Soc., Ser. B, 26:398-456, 1964.

[LP94] M. Loretan and P.C.B. Phillips. Testing the covariance stationarity of heavy-tailed time series. J. Empirical Finance, 1:211-248, 1994.

[Man63] B.B. Mandelbrot. The variation of certain speculative prices. Journal of Business, 26:394-419, 1963.

[Man82] B.B. Mandelbrot. The Fractal Geometry of Nature. W.H. Freeman and Co., San Francisco, 1982.

[McC94] J.H. McCulloch. Measuring tail thickness in order to estimate the stable index $\alpha$ : a critique. 1994. Preprint.

[MR93] S. Mittnik and S.T. Rachev. Modeling asset returns with alternative stable distributions. Economics Reviews, 1993. 
[Res87] S.I. Resnick. Extreme Values, Regular Variation and Point Processes. SpringerVerlag, New York, 1987.

[Sam65] P. Samuelson. Rational theory of warrant pricing. Industrial Management Review, 6:13-32, 1965.

[Spa93] A. Spanos. On modeling speculative prices: Student's $t$ autoregressive model with dynamic heteroskedasticity. 1993. Technical report, University of Cyprus.

[Tay86] S.J. Taylor. Modelling Financial Time Series. Wiley, New York, 1986.

[Ver70] D. Vere-Jones. Stochastic models for earthquake occurencies. Journal Royal Stat. Soc., Ser. B, 532:1-42, 1970.

Gennady Samorodnitsky

School of Operations Research and Industrial Engineering

Cornell University

Ithaca, NY 14853

U.S.A. 